\title{
The effect of network size and density to the choice of zone radius in ZRP
}

\author{
Hussein M. Haglan ${ }^{1}$, Salman Yussof ${ }^{2}$, Khalid W. Al-Ani ${ }^{3}$, Hothefa Shaker Jassim ${ }^{4}$, Dhamea A. Jasm ${ }^{5}$ \\ ${ }^{1}$ Computer Center, University of Anbar, Iraq \\ ${ }^{2}$ College of Computer Science and Information Technology, Universiti Tenaga Nasional, Malaysia \\ ${ }^{3}$ Presidency University, University of Anbar, Iraq \\ ${ }^{4}$ Modern College of Business and Science, Oman \\ ${ }^{5}$ Directorate of Education in Anbar, Ministry of Education, Iraq
}

\begin{tabular}{l}
\hline Article Info \\
\hline Article history: \\
Received Feb 3, 2020 \\
Revised Apr 4, 2020 \\
Accepted Apr 18, 2020 \\
\hline
\end{tabular}

\section{Keywords:}

Hybrid routing

MANET

Proactive routing

Reactive routing

Zone routing protocol

\begin{abstract}
Mobile Ad hoc Network (MANET) is a network that consists of several nodes that connect without using a permanent infrastructure. Each node in MANET moves inside and outside of the network freely and randomly. The free and random movements of nodes may cause the topology of the network to change constantly. Therefore, the task of finding routes between nodes is a big challenge. Routing protocols in MANET can be divided into three categories, namely, proactive, reactive and hybrid routing protocols. Hybrid routing protocols such as the Zone Routing Protocol (ZRP) combines the advantages of both proactive and reactive routing protocols by dividing the network area into many overlapping zones. Data transmission to nodes within the zone is done using a proactive routing mechanism, while data transmission to nodes outside the zone is done using a reactive routing mechanism. The zone radius in ZRP determines how much proactive and reactive routing is used, and therefore plays a key role in determining the performance of the network. In this paper, the performance of the ZRP routing protocol is evaluated using the NS2.33 network simulator. The focus of the research is to evaluate the ZRP performance concerning the network size and density to identify an optimum ZRP zone size that will provide good performance. The performance is evaluated by using four performance metrics which are normalized routing load, average end-to-end delay, throughput, and packet delivery fraction. The results show that ZRP produces the best performance when the zone radius size is equal to two hops.
\end{abstract}

Copyright (c) 2020 Institute of Advanced Engineering and Science. All rights reserved.

\section{Corresponding Author:}

Hussein M. Haglan,

Computer Center,

University of Anbar, Ramadi, Anbar, Iraq.

Email: hussein.m.haglan@uoanbar.edu.iq

\section{INTRODUCTION}

A MANET is a collection of wireless nodes that can dynamically form a network to exchange information without using any pre-existing fixed network infrastructure [1-3]. Each node in MANET uses its transmission range to send and receive packets $[4,5]$. Routing paths in mobile ad-hoc networks potentially contain multiple hops and some nodes are unable to communicate directly with other remote nodes because the transmission range of each node in the MANET is limited. Therefore, the responsibility of every node in MANETs is to work as a router [6,7]. Designing routing algorithms for MANET imposes a big challenge $[8,9]$. One of the reasons is because the random mobility of nodes in MANET can affect the network topology through frequent topology changes and network partitions. Another reason is that the variable and unpredictable capacity and availability of wireless links may cause a large amount of packet loss. Moreover, 
the broadcast nature of the wireless medium introduces the hidden terminal and exposed terminal problems. Additionally, mobile nodes have restricted power, computing and bandwidth resources and require effective routing schemes. Therefore, the design of an optimum routing protocol for MANET is highly complex $[10,11]$.

There are three categories of MANET routing protocols: proactive, reactive and hybrid. In a proactive routing protocol, each node maintains an up-to-date routing table. In a reactive routing protocol, a new route is created based on demand. Although proactive routing protocols provide high reliability and low latency in finding a route, these protocols do not perform well in a large MANET because of the overhead required to maintain an up-to-date routing table for thousands of mobile nodes. This large routing overhead can decrease the total bandwidth efficiency. Reactive routing protocols, on the other hand, do not need to maintain an up-to-date routing table for the whole MANET. Routes are acquired on-demand. However, this may cause a significant delay every time a new route is requested, which in turn can reduce the performance in real-time traffics. Hybrid routing protocols attempt to combine the best features of proactive and reactive protocols [12-14]. In hybrid routing protocols, each node will keep an up-to-date routing table only to a group of other nodes. This will allow data transmission to these nodes to be done instantly, without the delay to search for a path. On the other hand, since the routing table only keeps up-to-date routes to a small group of nodes, routing overhead can be significantly reduced. An example of a hybrid routing protocol is the Zone Routing Protocol (ZRP) [15-18].

In ZRP, the number of nodes involved in proactive routing is determined by the zone size. The larger the zone size, the larger the number of nodes that a node needs to have an up-to-date path to in its routing table. Although this will theoretically decrease the delay in sending data to these nodes, having more nodes in the up-to-date routing table may also increase the control packets being sent to maintain the routing table, which in turn may congest the network and degrade the routing performance in a certain way. As a result, there is a limit in terms of the number of nodes that should be involved in proactive routing. Therefore, it is important to identify an optimum ZRP zone size that will provide good performance concerning different routing performance metrics. In this paper, the optimum ZRP zone size will be determined using simulation. In the simulation, multiple MANETs with various network size and density will be evaluated with different values of zone radius.

The zone radius in ZRP is an important parameter because it determines the zone size and thus, it can satisfy the balance between proactive routing and reactive routing and provide an optimum routing performance for ZRP. Jagga et al. [19], three MANET routing protocols were evaluated, which are DSR, STAR, and ZRP concerning throughput, jitter, and average end-to-end delay. The result shows that STAR was better than DSR and ZRP. Pathak et al. (2015) [20] evaluated the performance of three routing protocols in MANET which are AODV and DSR as reactive routing protocols, and ZRP as a hybrid routing protocol, for the packet size. The result shows that DSR is better than AODV and ZRP when the packet size is small, while AODV is best when the packet size is large. Dixit and Shrivastava [21] evaluated the performance of three MANET routing protocols which are DSR, AODV, and ZRP by using directional metamaterial antenna. The result shows that DSR is better than AODV and ZRP.

As a summary of the relevant works reviewed above, many researchers in the recent past years have been done many kinds of research on ZRP but few of them have researched the effect of zone radius in ZRP on network performance. In particular its effect on the network performance to the nodes density and the network size. In ZRP, the density of the nodes involved in proactive routing is determined by the zone size. The larger the zone size, the larger the number of nodes that a node needs to have an up-to-date path to in its routing table. Although this will theoretically decrease the delay in sending data to these nodes, having more nodes in the up-to-date routing table may also increase the control packets being sent to maintain the routing table, which in turn may congest the network and degrade the routing performance in a certain way. As a result, there is a limit in terms of the number of nodes that should be involved in proactive routing. Therefore, they need to know the suitable zone radius is very important and its impact on the two most important metrics in the network, which are the network size and density. In this paper, the optimum ZRP zone size will be determined using simulation. In the simulation, multiple MANETs with various network sizes and density will be evaluated with different values of zone radius.

\section{SIMULATION SETUP}

Two different scenarios are created and simulated in the NS2.33 simulator to evaluate the performance of the ZRP routing protocol. The difference between the two scenarios lies in the simulation parameters which are to be evaluated. In the first scenario, the performance of the ZRP routing protocol is evaluated concerning the number of nodes in the network. Increasing the number of nodes in a network of a specific size will increase the network density. In the second scenario, the performance of the ZRP routing 
protocol is evaluated for the network size. In each scenario, four test cases are evaluated. In the first test case, the zone radius was configured to be two hops. For the subsequent test cases, the zone radius was increased to three, four and five respectively. The performance of the four test cases in each scenario is compared with each other to identify the zone radius that gives the best routing performance.

In the simulation, nodes are free to move arbitrarily; thus, the network topology that is typically multi hops may change randomly and rapidly at unpredictable times. In scenario 1, the number of nodes was varied and they were distributed within a $1500 \mathrm{~m} \times 1500 \mathrm{~m}$ area. In scenario 2, the density of the nodes was constant to 50 nodes. These nodes were spread inside a network environment of various sizes. In both scenarios, the simulation period was equal to 900 seconds, the radio range was set to 250 meters, the pause time was constant to 20 second and the maximum speed was $20 \mathrm{~m} / \mathrm{s}$. The simulation parameters for the two scenarios are shown in Table 1.

Table 1. Simulation parameters for scenarios 1 and 2

\begin{tabular}{lll}
\hline Simulation Parameters & Scenario 1 & Scenario 2 \\
\hline Number of Nodes & $50,60,70,80,90$ and 100 nodes & 50 nodes \\
Simulation Time & 900 seconds & 900 seconds \\
Map Size & $1500 \mathrm{~m} \mathrm{x} 1500 \mathrm{~m}$ & $(1500 \mathrm{~m} \mathrm{x} \mathrm{1500m)}-(2250 \mathrm{~m} \times 2250 \mathrm{~m})$ \\
Max Speed & $20 \mathrm{~m} / \mathrm{s}$ & $20 \mathrm{~m} / \mathrm{s}$ \\
Mobility Model & Random waypoint & Random waypoint \\
Traffic Type & Constant bit rate $(\mathrm{CBR})$ & Constant bit rate $(\mathrm{CBR})$ \\
Packet Size & 512 bytes & 512 bytes \\
Connection Rate (Nominal Radio Range) & 4 pkts/sec & 4 pkts/sec \\
Pause Time & $20 \mathrm{~second}$ & 20 second \\
Number of Connection & 5 & 5 \\
Bandwidth of links & $2 \mathrm{Mbit}$ & $2 \mathrm{Mbit}$ \\
MAC layer type & IEEE 802.11 & IEEE 802.11 \\
Radio range & 250 & 250 \\
\hline
\end{tabular}

\section{PERFORMANCE METRICS}

The performance metrics used are the standard metrics commonly used to measure and evaluate the performance of routing protocols. In this research, four performance metrics are used. The performance metrics selected are:

Packet Delivery Fraction (PDF): it is to measure the success of the protocol performance in delivering packets sent by the source node to the target node. Through which the ratio of the number of packets received to the target node divided by the number of data packets sent from the source node. The higher the result value is the better the protocol performance, the higher the value is the best in delivering the data to the target node. This metric calculates the correctness, completeness, and reliability of the routing protocol performance to measure its effectiveness. The following equation has used to calculate the packet delivery fraction (PDF) in the performance of ZRP [22-24]:

$$
P D F=\frac{\sum_{m=1}^{n} \text { recvs }}{\sum_{m=1}^{n} \text { sends }} \times 100
$$

Throughput: it is to measure the ratio of the number of data packets that have successfully received by the target node over a specified period (bytes per second). Factors that affect the throughput include frequent changes in the network topology, limited bandwidth, unreliable communication, and limited energy. The following equation has used to calculate the throughput in performance of ZRP [2, 25]:

$$
\text { Throughput }=\frac{\sum_{m=1}^{n} \text { recv }}{\text { SimulationTime }}
$$

Average End-to-End Delay: it is to measure the total delay occurring during the packet data journey from the source node to the target node. Collecting several small delays produces a total delay in the network. These delays include all the delays that are likely to occur during the packet journey such as waiting until the path is found, waiting in the queue until it reaches its role in the transportation, delayed production at the failure of transport and re-transmission. The average end-to-end delay (E2E) metric depicts the packet journey time from the source node to the target node: Whenever the average end-to-end delay is lower will be the best performance. It is calculated through dividing the total time differences between the send times of CBR packets and their receive times on the total number CBR packets that have received. The following equation has used to calculate the average E2E of data packets in the performance of ZRP [5, 26]. 


$$
E 2 E=\frac{\sum_{m=1}^{n}(C B R \text { Sent } \text { Time }-C B R \text { Recv Time })}{\sum_{m=1}^{n} \text { reconum }}
$$

Normalized Routing Load: it is a calculated number of control messages that have sent for routing in the network to determine the load on the network. It is measured by calculating the number of routing messages sent per data packet has reached the target node. Each hop crossed by the routing message during the transportation is considered as one transmission. The following equation has used to calculate NRL [4, 27].

$$
N R L=\frac{\sum_{m=1}^{n} \operatorname{RPgen}}{\sum_{m=1}^{n} \text { recvs }}
$$

\section{SIMULATION RESULTS AND ANALYSIS FOR SCENARIO 1}

The packet delivery fraction of the four test cases of ZRP for scenario 1 is shown in Figure 1. The figure shows that the packet delivery fraction slightly decreases in all ZRP cases with an increase in the number of nodes. One possible reason that causes this decrease is that whenever the number of nodes increases, there will be more control messages due to the increase in the number of nodes within the zone, where they will use proactive mechanism. This can congest the network and therefore less network capacity is available for actual data delivery.

When comparing between those four test cases of ZRP, it is evident from Figure 1 that the packet delivery fraction of ZRP decreases gradually with the increase in zone radius as represented in the four cases of ZRP (i.e. The packet delivery fraction gradually decreases from the case that has zone radius equal to two to the cases that have larger zone radius). In ZRP, routing to nodes within the zone is done using IARP (proactive) and routing to nodes outside the zone is done using IERP (reactive). However, as the zone radius increases, the proactive area of the ZRP also increases. The nature of proactive routing protocol is that the nodes will flood the network with control messages to find new routes to nodes that belong to their routing zones, and update their routing tables whenever any change happens in the network topology because of the movements of the nodes. Therefore, the overhead and consumption of bandwidth are increased due to the increase in control messages. This negatively affects network performance. Therefore, it can be concluded that with the gradual increase in the size of the zone radius, the packet delivery fraction decreases.

The result of the simulation in terms of throughput is shown in Figure 2. Based on the results, it can be seen that the throughput slightly decreases with the increase in the number of nodes. The reason for this is similar to the reason that causes the decrease in the packet delivery fraction where whenever the number of nodes increases, there will be more control messages due to the increase in the number of nodes within the zone. This can congest the network and therefore less network capacity is available for actual throughput.

When comparing between those four cases of ZRP, it is evident from Figure 2 that the throughput of ZRP decreases gradually with the increase of zone radius. The reason is also the same reason that has caused the gradual decline in terms of the packet delivery fraction as mentioned above where the increase in zone radius will cause more control messages that will use a large amount of bandwidth. This, in turn, will decrease the throughput of data transmission. Therefore, it can be concluded that with the gradual increase in the size of the zone radius, the data throughput will also decrease.

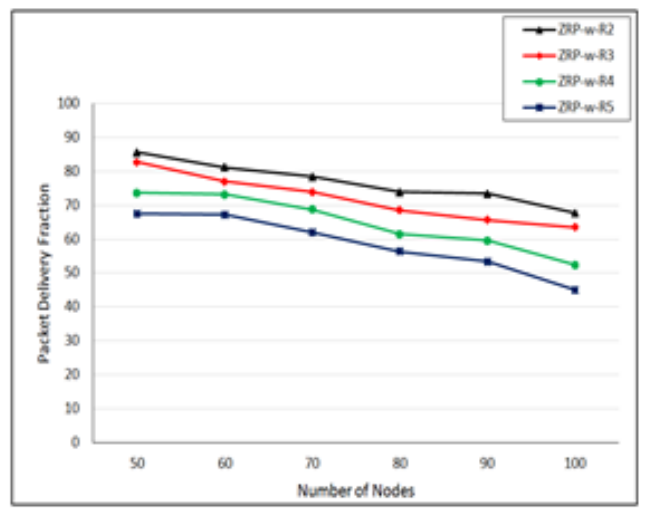

Figure 1. Packet delivery fraction for scenario 1

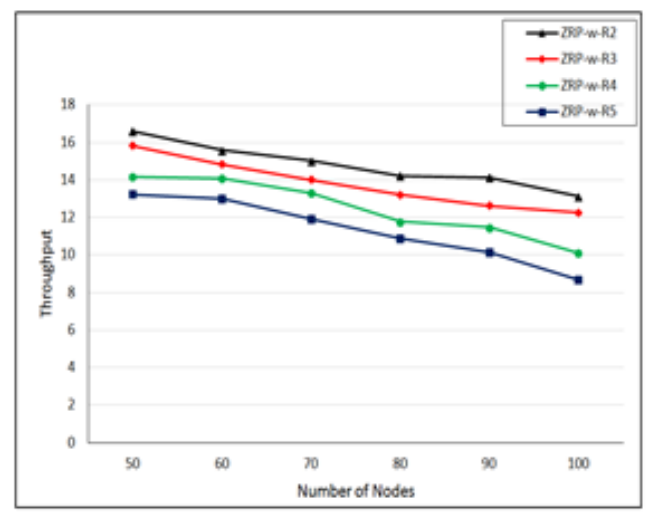

Figure 2. Throughput for scenario 1 
Figure 3 shows the result of the simulation in terms of the average end-to-end delay. It is evident that with the gradual increase in the number of nodes in the network, the average end-to-end delay also decreases regardless of the size of the zone radius. The reason is that with the increase in the number of nodes within the same network area, there will be more overlapping zones that will increase the use of IAPR, which uses proactive routing. With proactive routing, the routes are readily available and can be used immediately. This, in turn, will lower the average end-to-end delay in transmitting data from source to destination node regardless of the size of the zone radius. When comparing the four test cases as shown in in the figure that, it is evident that whenever there is a gradual increase in the size of the zone radius, the average end-to-end delay is gradually decreased. The last test case with the zone radius equals to five hops is better than all the other cases. The reason is due to the increased scope of IARP. With a larger radius, more nodes will be included in IARP, which uses proactive routing. Therefore, a larger portion of data transmission can be transmitted immediately without having to query for a path since the path is already available in the source node's routing table. As a result, as the zone size gets larger, the average end-to-end delay decreases.

Figure 4 shows the normalized routing load of the four test cases. From the figure, it is evident that the normalized routing load in all the four test cases is increasing gradually with the increase in the number of nodes. This is because increasing the number of nodes would consequently increase the number of control messages generated. Thus, the routing load of the nodes would also increase due to the need to process the increasing number of control messages. It also shows the normalized routing load as the zone radius gets larger. From the result, it can be seen that the larger the zone radius, the normalized routing load will also increase. The reason is that as the zone radius gets larger, more nodes will be included in IARP as a proactive manner; this will lead to more control messages. A summary of the results that have been obtained for scenario 1 is shown in Table 2 and Table 3.
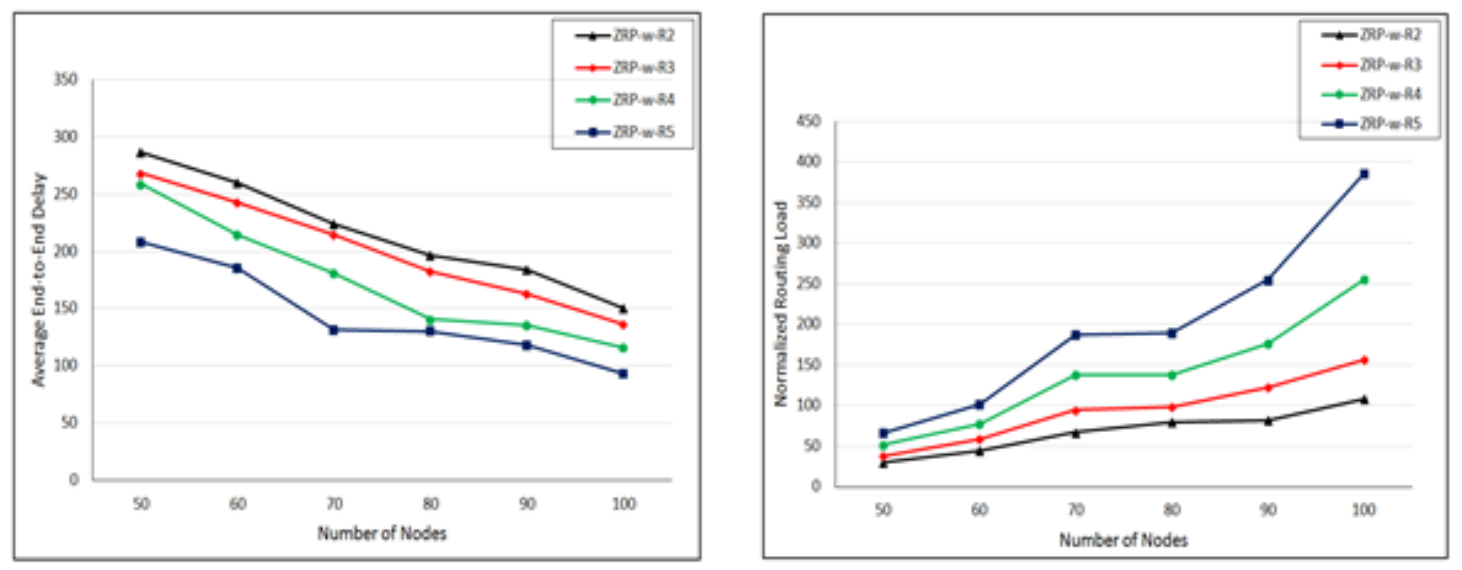

Figure 3. Average End-to-End delay for scenario 1 Figure 4. Normalized Routing Load for scenario 1

Table 2. Evaluation of the four test cases of ZRP for scenario 1

\begin{tabular}{ccccc}
\hline Performance Metrics & ZRP-w-R2 & ZRP-w-R3 & ZRP-w-R4 & ZRP-w-R5 \\
\hline PDF & 1 & 2 & 3 & 4 \\
Throughput & 1 & 2 & 3 & 4 \\
E2ED & 4 & 3 & 2 & 1 \\
NRL & 1 & 2 & 3 & 4 \\
\hline
\end{tabular}
sequentially.

Where (1), (2), (3), and (4) represent the best, the second-best, the third-best, and the worst,

Table 3. The effect of the number of nodes on ZRP performance for scenario 1

\begin{tabular}{cc}
\hline Performance Metrics & Variation in Performance Metrics When Nodes Density Increased in the Network \\
\hline PDF & Decrease \\
Throughput & Decrease \\
E2ED & Decrease \\
NRL & Increase \\
\hline
\end{tabular}




\section{SIMULATION RESULTS AND ANALYSIS FOR SCENARIO 2}

The result for packet delivery fraction is shown in Figure 5. In scenario 2, the packet delivery fraction gradually decreases in all test cases with the increase of the network size and continues to decline until they became very close to each other in a large network (2250m x 2250m).

There could be two reasons for this. The first reason is that as the network size increases, the nodes become distributed in a wide area, thus the distance between the nodes becomes farther and nodes have more freedom of movement. Therefore, the topology of the network becomes more prone to change. For this reason, the overhead is increased as the intermediate nodes send more control messages to update their routing tables using IARP inside their routing zones. Because of this overhead, the network becomes busy most of the time and this negatively affects the network performance. The second reason is that due to the long distance between nodes and the limited radio range of the nodes, the opportunities to provide routes between the source nodes and the destination nodes become lower. Therefore, it can be concluded that the packet delivery fraction decreases as the network size increases.

When comparing the four test cases as shown in Figure 5, it is evident that there is a gradual decline in terms of the packet delivery fraction whenever the value of the zone radius is increased. The reason that has led to the gradual decline in the packet delivery fraction as the zone radius gets larger is due to the increase in the overhead and bandwidth consumption whenever the size of the zone radius is increased. The overhead is a result of an increase in the use of IARP, which is flooding the network with control messages whenever there is a change in the network topology to identify new routes to all nodes within the zone and update their routing tables. Thus, the negative effect of the overhead on network performance is increased whenever the zone radius increases. Therefore, the first test case where the zone radius equals to two hops was the best in terms packet delivery fraction compared to the other test cases with larger zone radius because with smaller zone radius, the number of nodes involved in proactive routing is small and this lowers the utilization of bandwidth for control messages. On the other hand, when the zone radius is small, the area that uses the reactive routing mechanism (IERP) is wider than the area that uses the proactive routing mechanism (IARP). The reactive routing mechanism is used to find routes to nodes outside the zones on demand and the advantages for this routing protocol lie in its low overhead and low bandwidth consumption, and are therefore suitable for large networks. All these advantages of IERP are exploited when the zone radius size is small.

Figure 6 shows the simulation result in terms of throughput for scenario 2. The figure shows that the throughput in all four test cases decreases whenever the network size is increased and continues to decline until they became very close to each other in a large network. The reason that led to this decrease is the same reason that caused a gradual decline in terms of packet delivery fraction as explained above.

Figure 6 also shows that there is a gradual decline in terms of throughput whenever the value of the zone radius is increased. The first test case where the zone radius is equal to two hops is the best in terms of throughput. Again, the explanation of why this happens is similar to the explanation for the result of the packet delivery fraction above.

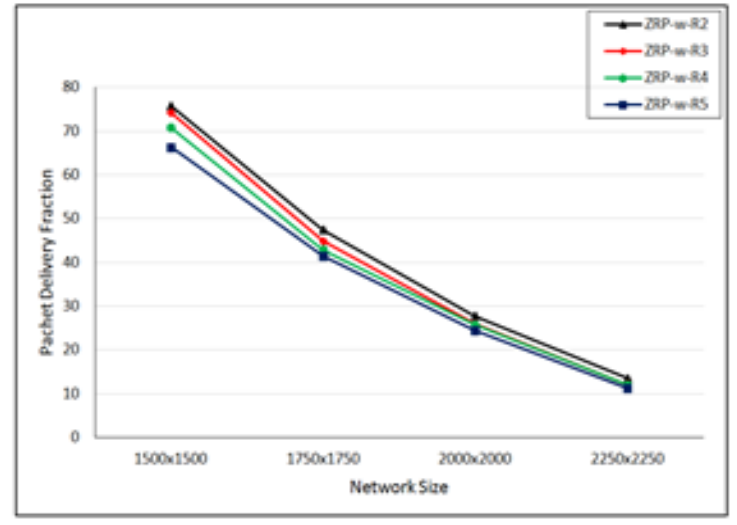

Figure 5. Packet delivery fraction for scenario 2

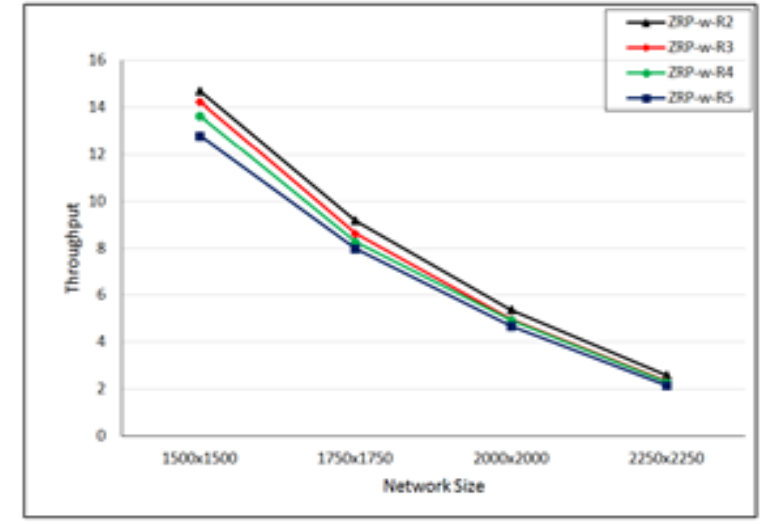

Figure 6. Throughput for scenario 2

Figure 7 shows the simulation result for the average end-to-end delay. Based on the result, it can be seen that as the network size gets larger, the delay is also getting longer. This is because as the network size gets larger, the distance between nodes may also be larger. Therefore, the propagation delay between nodes will increase. Furthermore, as the distance between nodes gets larger, some nodes may be inaccessible for a 
certain duration due to the limited radio range. The source node may require several attempts before those nodes can be found by the routing protocol and this will cause further delay.

Figure 8 shows the normalized routing load for the four test cases in scenario 2. This figure shows that the normalized routing load increases in all test cases whenever the network size is increased. The reason is that as the network size gets larger, nodes are free to move farther. This makes it easy for links to be broken and the nodes involved need to generate and process more control messages to update their routing tables. This increases the routing loads of the nodes. A summary of the results that have been obtained for scenario 2 is shown in Table 4 and Table 5.

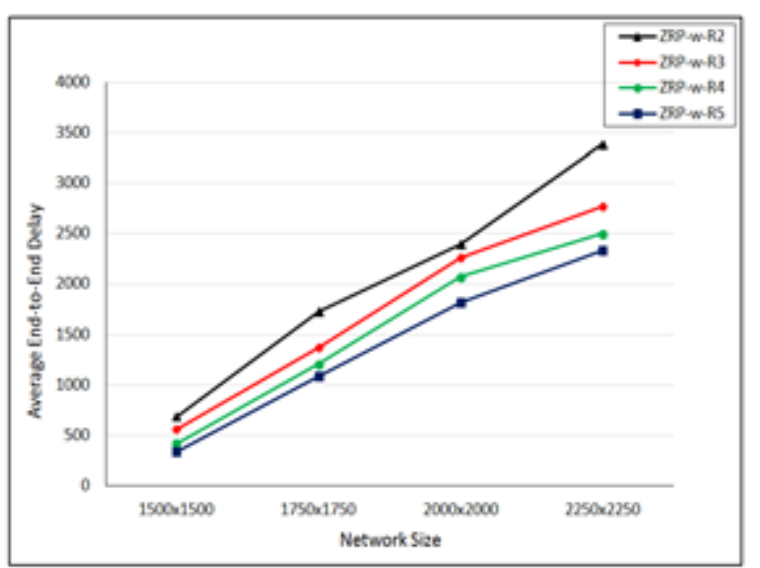

Figure 7. Average End-to-End Delay for scenario 2

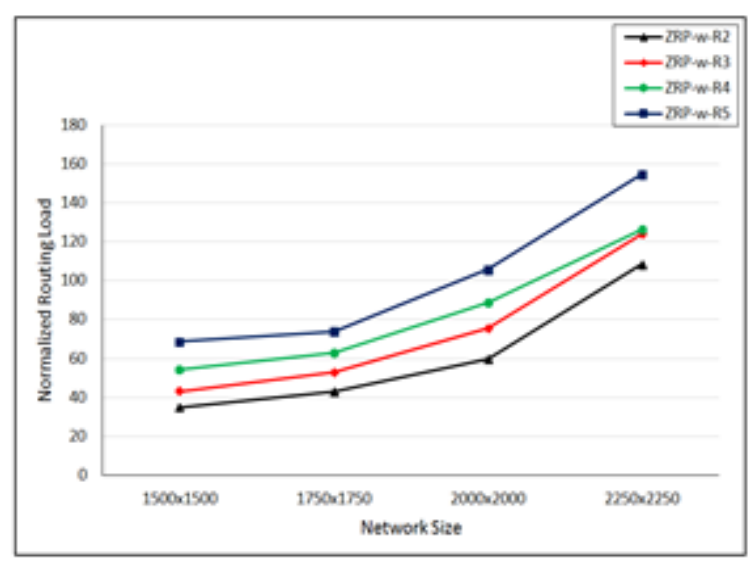

Figure 8. Normalized Routing Load for scenario 2

Table 4. Shows an evaluation to four test cases of ZRP for scenario 2

\begin{tabular}{ccccc}
\hline Performance Metrics & ZRP-w-R2 & ZRP-w-R3 & ZRP-w-R4 & ZRP-w-R5 \\
\hline PDF & 1 & 2 & 3 & 4 \\
Throughput & 1 & 2 & 3 & 4 \\
E2ED & 4 & 3 & 2 & 1 \\
NRL & 1 & 2 & 3 & 4 \\
\hline
\end{tabular}

Where (1), (2), (3), and (4) represent the best, the second-best, the third-best, and the worst.

Table 5. The effect of the network size on ZRP performance for scenario 2

\begin{tabular}{cc}
\hline Performance Metrics & Variation in Performance Metrics when Network Size increased \\
\hline PDF & Decrease \\
Throughput & Decrease \\
E2ED & Increase \\
NRL & Increase \\
\hline
\end{tabular}

\section{CONCLUSION}

The performance of ZRP has been examined and evaluated the NS2 Network Simulator to identify the zone radius that gives the best routing performance for ZRP. Two scenarios have been used in this simulation. The first scenario evaluates the network performance in terms of network density and the second scenario evaluates the performance in terms of network size. In each scenario, we examined four test cases, wherein each test case; a different zone radius value is used. The zone radius used for the test cases is 2, 3, 4 and 5 hops respectively. To evaluate network performance, four performance metrics are used. The performance metrics used are packet delivery fraction, throughput, average end-to-end delay, and normalized routing load. Based on the results given by the simulation, it is clear that an increase in either the number of nodes in the network or the network size will lower the performance of the network. It can also be seen that a zone radius of two hops provides the best performance for three out of the four performance metrics used in this research. The three performance metrics are packet delivery fraction, throughput and normalized routing load. Only network performance in terms of end-to-end delay becomes better as the zone size gets larger. 


\section{REFERENCES}

[1] M. H. Hassan, and R. C. Muniyandi, "An improved hybrid technique for energy and delay routing in mobile ad-hoc networks," International Journal of Applied Engineering Research, vol. 12, no. 1, pp. 134-139, 2017.

[2] M. Jubair, and R. Muniyandi, "NS2 simulator to evaluate the effective of nodes number and simulation time on the reactive routing protocols in MANET," International Journal of Applied Engineering Research, vol. 11, no. 23, pp. 11394-11399, 2016.

[3] M. H. Hassan, et al., "Integrating African Buffalo Optimization Algorithm in AODV Routing Protocol for improving the QoS of MANET," Journal of Southwest Jiaotong University, vol. 54, no. 3, 2019.

[4] M. A. Jubair, et al., "Performance Evaluation of Ad-Hoc On-Demand Distance Vector and Optimized Link State Routing Protocols in Mobile Ad-Hoc Networks," International Journal on Advanced Science, Engineering and Information Technology, vol. 8, no. 4, pp. 1277-1283, 2018.

[5] B. A. Khalaf, et al., "A Simulation Study of Syn Flood Attack In Cloud Computing Environment," AUS journal, vol. 26, pp. 1, pp. 188-197, 2020.

[6] M. A. Jubair, et al., "Bat Optimized Link State Routing Protocol for Energy-Aware Mobile Ad-Hoc Networks," Symmetry, vol. 11, no. 11, p. 1409, 2019.

[7] D. Bisen, et al., "Effect of pause time on DSR, AODV and DYMO routing protocols in MANET," International Journal of IT \& Knowledge Management, vol. 3, no. 1, 2010.

[8] M. A. Jubair, et al., "Competitive analysis of single and multi-path routing protocols in mobile Ad-Hoc network," International Journal of Electrical, Electronics and Computer Systems (IJEECS), vol. 19, no. 1, pp. 293-300, 2020.

[9] S. A. Mostafa, et al., "A Multi-Agent Ad Hoc On-Demand Distance Vector for Improving the Quality of Service in MANETs," International Symposium on Agent, Multi-Agent Systems and Robotics (ISAMSR), pp. 1-7. IEEE, 2018.

[10] K. Raheja and S. K. Maakar, "A Survey on Different Hybrid Routing Protocols of MANET," Int. J. Comput. Sci. Inf. Technol., vol. 5, no. 4, pp. 5512-5516, 2014.

[11] D. Ravilla, et al., "Hybrid routing protocols for ad hoc wireless networks," International Journal of Ad hoc, Sensor \& Ubiquitous Computing, vol. 2, no. 4, pp. 79-96, 2011.

[12] G. A. Walikar and R. C. Biradar, "A survey on hybrid routing mechanisms in mobile ad hoc networks," Journal of Network and Computer Applications, vol. 77, pp. 48-63, 2017.

[13] G. R. Chandra, "A new algorithm for pervasive computing in manet," Int. J. Eng. Sci. Adv. Technol., vol. 1, no. 1, pp. 43-46, 2011.

[14] R. K. Gujral, et al., "Impact of Scalability with respect to Mobility and Zone Size on Zone Routing Protocol over MANETs," International Conference on Computing Sciences (ICCS), IEEE, pp. 343-349, 2012.

[15] H. Xia, et al., "Applying link stability estimation mechanism to multicast routing in MANETs," Journal of Systems Architecture, vol. 60, no. 5, pp. 467-480, 2014.

[16] C. Liu and J. Kaiser, "A Survey of Mobile Ad Hoc network Routing Protocols," Technical Report, Department of Computer Structures, University of Ulm, Germany, 2005.

[17] Y. R. Kumar and S. k. Chittamuru, "A Case Study on MANET Routing Protocols Performance over TCP and HTTP," Master Thesis in Electrical Engineering, Thesis no: MSE-2010-6434, School of Engineering, Blekinge Institute of Technology, 2010.

[18] A. K. Pandey and H. Fujinoki, "Study of MANET routing protocols by GloMoSim simulator," Int. J. Netw. Manag., vol. 15, no. 6, pp. 393-410, 2005.

[19] S. Jagga, et al., "Performance Analysis of DSR, STAR, ZRP Routing Protocols for a Dynamic Ad-Hoc Network," Int. Res. J. Eng. Technol., vol. 4, no. 2, pp. 1428-1431, 2017.

[20] H. Pathak, et al., "Comparing the Item Size of Aodv, Dsr \& Zrp Routing Protocols in Mobile Ad Hoc Network Using Qualnet Simulator 5.0.2," Int. J. Eng. Sci. Res., vol. 5, no. 7, pp. 754-759, 2015.

[21] A. Dixit and L. Shrivastava, "Performance Analysis Of Aodv, Dsr And Zrp Routing Protocols In Manet Using Directional Antenna," Int. Res. J. Eng. Technol., vol. 2, no. 7, pp. 216-221, 2015.

[22] G. Anastasi, E. Borgia, M. Conti, and E. Gregori, "IEEE 802. 11 Ad Hoc Networks : Performance Measurements," International Conference on Distributed Computing Systems Workshops, IEEE, pp. 758-763, 2003.

[23] N. Z. M. Safar, et al., "Characterising and detection of botnet in P2P network for UDP protocol," Indonesian Journal of Electrical Engineering and Computer Science (IJEECS), vol. 18, no. 3, 1584-1595, 2020.

[24] A. El Aalaoui, et al., "Energy efficiency of organized cluster election method in wireless sensor networks," Indonesian Journal of Electrical Engineering and Computer Science (IJEECS), vol. 18, no. 1, pp. 218-226, 2020.

[25] E. Tuba et al., "Wireless sensor network coverage problem using modified fireworks algorithm," International Wireless Communications and Mobile Computing Conference (IWCMC), IEEE, pp. 696-701, 2016.

[26] F. Bai and A. Helmy, "A Survey of Mobility Models," in Wireless Ad hoc Wireless Ad-Hoc Networks, Kluwer Acad. Chapter 1. Last accessed March 2012, 2006.

[27] S. Yussof, T. S. Kiong, S. P. Koh, and R. Ismail, "A routing protocol based on trusted and shortest path selection for mobile ad hoc network," IEEE 9th Malaysia International Conference on Communications (MICC), pp. 547-554, 2009. 